NISSUNA UMANA INVESTIGAZIONE SI PUO DIMANDARE VERA SCIENZIA S'ESSA NON PASSA PER LE MATEMATICHE DIMOSTRAZIONI LEONARDO DA VINCI

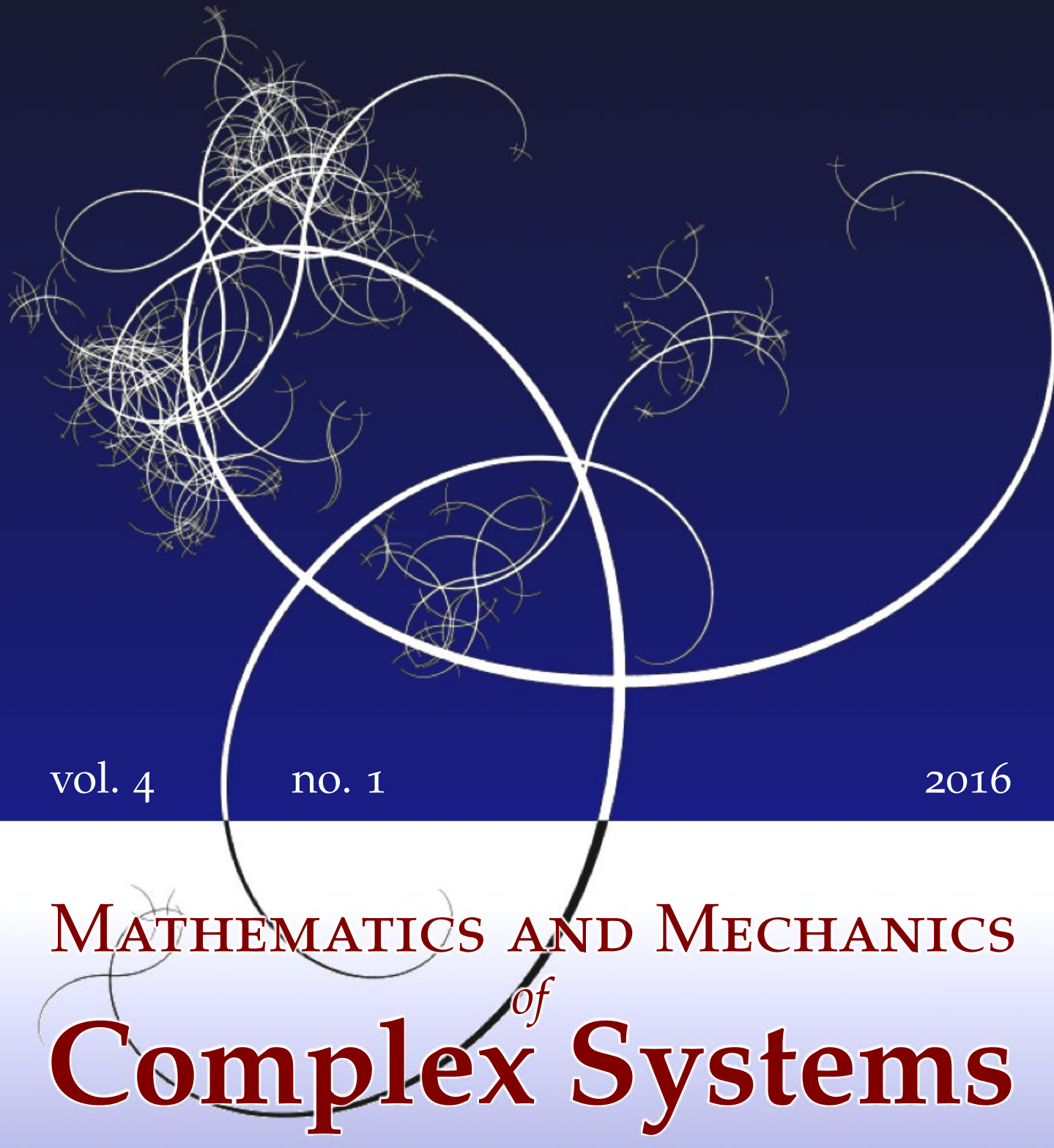

GÉRARd GAGNeuX AND Olivier Millet MODELING CAPILLARY HYSTERESIS IN UNSATURED POROUS MEDIA 


\title{
MODELING CAPILLARY HYSTERESIS IN UNSATURED POROUS MEDIA
}

\author{
GÉRARd GAGneuX AND Olivier Millet
}

\begin{abstract}
This paper deals with the modeling of cyclic hysteresis phenomena for flows in unsaturated porous media, using a dynamic regularization process of Sobolev type. The addition of a kinematic regularizing term of third-order partial derivatives, depending on a strictly positive, small real parameter, enables us to capture the missing information of the ill-posed hysteresis phenomena via Rankine-Hugoniot and "entropy" inequalities. When this parameter tends to zero, an oriented hysteresis loop, corresponding to the realistic problem modeled, emerges from the flow of an associated auxiliary ordinary differential equation.
\end{abstract}

\section{Introduction}

The modeling of moisture transport in partially saturated porous media is of major importance for civil engineering, soil physics, and pharmaceutical applications. The hysteresis effects, often neglected in the modeling as they are difficult to be taken into account, play a central role in the imbibition and drying process.

In this paper, we propose an original modeling of cyclic hysteresis phenomena in partially saturated porous media, in the simplified case of water-air flows. The approach used is based on the artificial introduction of an unstable spinodal interval and on Sobolev's method of dynamic regularization, inspired by the works of P. I. Plotnikov [1996; 1994], publicized by L. C. Evans and M. Portilheiro [2004; Evans 2004]. The hysteresis graph is replaced by Cartesian curves and an artificial spinodal interval generating instabilities, with associated attractive-repulsive dynamics.

The additional information to describe the hysteresis effects is introduced on the form of entropy-type inequalities. This way, the asymptotic limit of viscous approximate solutions generates effects of irreversibility and enables us to recover the expected hysteresis loop.

\section{Communicated by Francesco dell'Isola.}

MSC2010: primary 35K65; secondary 47J40, 76S05.

Keywords: capillary hysteresis loop, unsaturated porous media. 


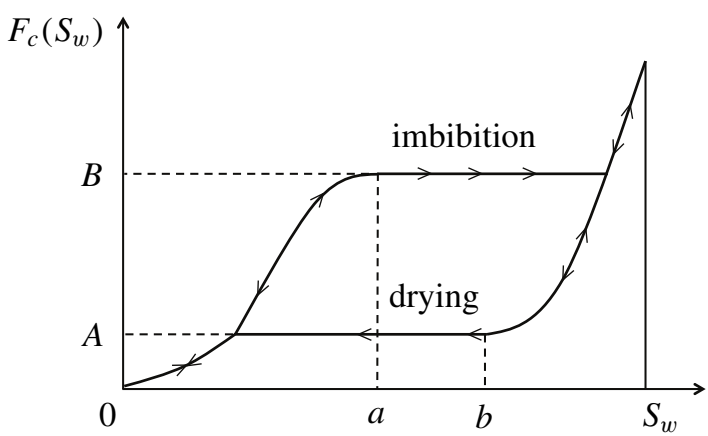

Figure 1. Graph of $F_{c}$ representing the hysteresis loop during an imbibition-drying process.

\section{The physical problem}

2.1. Richards equation. The flow of two fluid phases (water-air flow), isothermal and immiscible, in an unsaturated porous medium is considered. To focus on the study of the hysteresis effects, in particular irreversibility, the gravity is neglected and the porous medium is assumed to be homogeneous and isotropic. ${ }^{1}$ Moreover, the water vapor in the gas phase is neglected and the air pressure is assumed to be constant and equal to the atmospheric pressure. ${ }^{2}$

The water saturation $S_{w}$ is classically governed by a Richards equation,

$$
\varphi \frac{\partial S_{w}}{\partial t}-\Delta \Theta_{c}\left(S_{w}\right)=0,
$$

where $\varphi$ denotes the porosity of the porous medium considered. We assume that the residual saturation of each fluid is equal to zero.

Even if the mathematical analysis of this equation is now well stated [Gagneux and Madaune-Tort 1995; Lions 1969], it ignores the hysteresis and dynamic effects that play a major role in the behavior of unsaturated porous media.

2.2. Hysteresis modeling. The capillary hysteresis effects can be modeled with a multivalued operator $F_{c}$ whose oriented graph $F_{c}$ of $\mathbb{R}^{2}$ is represented in Figure 1 . The circulation sense depends both on the values of $S_{w}$ and on the sign of $\frac{\partial S_{w}}{\partial t}$. It characterizes the imbibition and drying phases, through the differential inclusion ${ }^{3}$

$$
0 \in\left\{\varphi \frac{\partial S_{w}}{\partial t}-\Delta F_{c}\left(S_{w}, \operatorname{sign}\left(\frac{\partial S_{w}}{\partial t}\right)\right)\right\} .
$$

\footnotetext{
${ }^{1}$ The analysis holds also for the anisotropic case.

${ }^{2}$ It is equivalent to assume that the gas phase moves fast and is connected to outside.

${ }^{3}$ In the sense of [Aubin and Cellina 1984].
} 


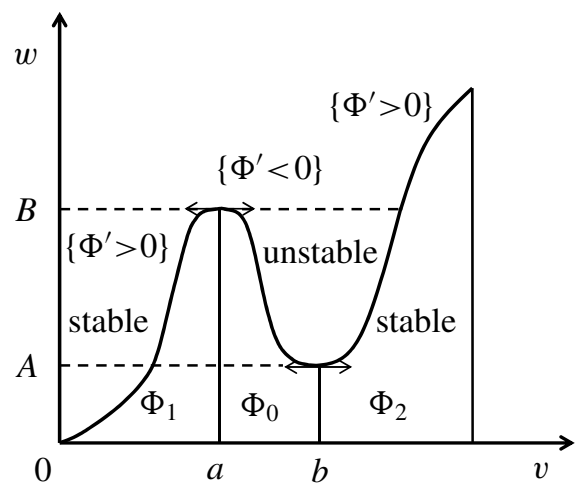

Figure 2. The spinodal interval $[a, b]$ and the unstable part of the graph of $\Phi$.

It is equivalent to search a pair $\left(S_{w}, q_{w}\right)$ with $q_{w} \in\left\{F_{c}\left(S_{w}, \operatorname{sign}\left(\frac{\partial S_{w}}{\partial t}\right)\right)\right\}$ which satisfies

$$
\varphi \frac{\partial S_{w}}{\partial t}-\Delta q_{w}=0
$$

associated to a Cauchy initial condition $S_{w(0)}$ and Neumann homogeneous boundary conditions. To simplify the problem without loss of generality, we will consider in the following a normalized porosity $\varphi=1$ (this is always possible using a homothetical time scaling, when $\varphi$ is constant).

In a first step, using a mathematical artifice, we replace the part of the graph $F_{c}$ representing the loop by a cubic Cartesian curve $\Phi$ (Figure 2). Then, to approach problem $\left(\mathscr{P}_{\text {hyst }}\right)$, a nonlinear monotone diffusion equation with an ad hoc "spinodal" interval $] a, b$ [is introduced. The graph $F_{c}$ is replaced on $] a, b$ [ by a cubic spline function, denoted $\Phi_{0}$, whose slope is strictly negative everywhere on $] a, b$ [ and assuring a $\mathscr{C}^{1}$ continuity at $(a, B)$ and $(b, A)$ with the preserved part.

The graph $F_{c}$ is decomposed by splitting its domain of definition into three distinct parts. This leads us to introduce three injective functions, $\Phi_{0}, \Phi_{1}$ and $\Phi_{2}$, defined on $[a, b],[0, a]$ and $[b, 1]$, respectively. We denote by $\beta_{0}, \beta_{1}$ and $\beta_{2}$ their respective inverse functions and by $\Phi$ the numerical function of class $\mathscr{C}^{1}$ on $[0,1]$ whose graph is the joining of the graphs of $\Phi_{0}, \Phi_{1}$ and $\Phi_{2}$.

This substitution enables us to give sense to the initial formal problem in a suitable mathematical functional framework, via the initial-boundary value system

$$
\begin{cases}\frac{\partial v}{\partial t}-\Delta \Phi(v)=0 & \text { in } Q=] 0, T[\times \Omega, \\ \frac{\partial \Phi(v)}{\partial n}=0 & \text { on } \Sigma=] 0, T[\times \Gamma, \\ v(0)=S_{w(0)} & \text { in } \Omega,\end{cases}
$$


where $\Omega$ denotes a bounded domain of $\mathbb{R}^{d}, d \geq 1$, with a Lipschitz boundary $\Gamma$ and an associated external unit normal vector $n$. The new forward-backward problem $\left(P_{\Phi}\right)$ with variable parabolicity direction is ill posed without any supplementary information, because of the nonmonotonic function $\Phi$. The dynamic regularization process that follows will enable us to regularize the problem. Note that a problem similar to $\left(P_{\Phi}\right)$ has been studied in [Smarrazzo and Tesei 2010; 2012; Smarrazzo 2008].

\section{Dynamic regularization process of Sobolev type}

The classical notations that follow are introduced. Let $\Omega$ be a bounded domain of $\mathbb{R}^{d}$ whose boundary $\Gamma$ is a Lipschitz manifold of dimension $d-1$. For $T>0$, we write $Q=] 0, T[\times \Omega, \Sigma=] 0, T\left[\times \Gamma\right.$ and let $\Delta$ be the Laplacian operator ${ }^{4}$ of $\mathbb{R}^{d}$. We denote by $H^{s}(\Omega), s \in \mathbb{R}$, the classical Hilbert spaces [Lions and Magenes 1968]. For all $\varepsilon>0$, the embedding of $H^{s}(\Omega)$ into $H^{s-\varepsilon}(\Omega)$ is compact. Moreover, we identify $L^{2}(\Omega)=H^{0}(\Omega)$ to its dual, so that the dual of $H^{1}(\Omega)$, denoted $H^{1}(\Omega)^{\prime}$, can be identified to an superspace of $L^{2}(\Omega)$ with $H^{1}(\Omega) \hookrightarrow L^{2}(\Omega) \hookrightarrow H^{1}(\Omega)^{\prime}$, the embeddings being dense and continuous. In addition, an initial state $S_{w(0)} \in L^{\infty}(\Omega)$ satisfying $0 \leq S_{w(0)} \leq 1$ a.e. in $\Omega$ is given.

The dynamical regularization process of Sobolev type used is based on "artificial viscosity". A parameter $\lambda>0$ is introduced in the initial ill-posed problem $\left(P_{\Phi}\right)$, which is transformed into the third order boundary problem

$$
\begin{cases}\frac{\partial v_{\lambda}}{\partial t}-\Delta \Phi\left(v_{\lambda}\right)-\lambda \Delta \frac{\partial v_{\lambda}}{\partial t}=0 & \text { in } Q=] 0, T[\times \Omega, \\ \frac{\partial}{\partial n}\left(\Phi\left(v_{\lambda}\right)+\lambda \frac{\partial v_{\lambda}}{\partial t}\right)=0 & \text { on } \Sigma=] 0, T[\times \Gamma, \\ v_{\lambda}(0)=S_{w(0)} & \text { in } \Omega .\end{cases}
$$

We then introduce the auxiliary dynamical unknown $w_{\lambda}$ defined by

$$
w_{\lambda}=\Phi\left(v_{\lambda}\right)+\lambda \frac{\partial v_{\lambda}}{\partial t},
$$

or equivalently

$$
\frac{\partial v_{\lambda}}{\partial t}=\frac{w_{\lambda}-\Phi\left(v_{\lambda}\right)}{\lambda}, \quad t>0 .
$$

Note that the dynamics created by (4) drive the system onto stable parts of the graph, as we will see in the sequel.

\footnotetext{
${ }^{4}$ In the sense of distributions.
} 
Problem $\left(P_{\Phi}\right)_{\lambda}$ may be rewritten as

$$
\begin{cases}\frac{\partial v_{\lambda}}{\partial t}-\Delta w_{\lambda}=0 & \text { in } Q=] 0, T[\times \Omega, \\ \frac{\partial w_{\lambda}}{\partial n}=0 & \text { on } \Sigma=] 0, T[\times \Gamma, \\ v_{\lambda}(0)=S_{w(0)} & \text { in } \Omega .\end{cases}
$$

Equivalently, for nearly all $t, w_{\lambda}$ is a solution of the elliptic problem parametrized in time ${ }^{5}$

$$
\begin{cases}w_{\lambda}-\lambda \Delta w_{\lambda}=\Phi\left(v_{\lambda}\right) & \text { in } \Omega, t>0, \\ \frac{\partial w_{\lambda}}{\partial n}=0 & \text { on } \Gamma, t>0 .\end{cases}
$$

The "- Laplacian" operator (denoted again $-\Delta$ ) specifically associated with homogeneous Neumann boundary conditions on $\Gamma$ is obviously a nonbounded operator from $L^{2}(\Omega)$ into $L^{2}(\Omega)$ whose domain is $H^{2}(\Omega)$. We introduce the Yosida regularization $-\Delta_{\lambda}:=-\Delta\left(J_{\lambda}\right)=\left(I-J_{\lambda}\right) / \lambda$ and its resolvent $J_{\lambda}=(I-\lambda \Delta)^{-1}$. According to (6), it follows that

$$
w_{\lambda}=J_{\lambda} \Phi\left(v_{\lambda}\right) .
$$

Problem $\left(P_{\Phi}\right)_{\lambda}$ can be formulated again in a well-posed form ${ }^{6}$ for the operator $-\Delta_{\lambda} \Phi$ in $L^{2}(\Omega)$ :

$$
\begin{cases}\frac{\partial v_{\lambda}}{\partial t}-\Delta_{\lambda} \Phi\left(v_{\lambda}\right)=0, & t \in] 0, T[ \\ v_{\lambda}(0)=S_{w(0)} & \text { in } L^{2}(\Omega) \text { and a.e. in } \Omega .\end{cases}
$$

The following proposition summarizes the properties of the solutions of $\left(P_{\lambda}\right)$ :

Proposition 1. Let us denote by $g: \mathbb{R} \rightarrow \mathbb{R}$ a Lipschitz nondecreasing function, a so-called "entropy function", and let

$$
G(r)=\int_{0}^{r} g(s) d s, \quad G_{\Phi}(r)=\int_{0}^{r} g(\Phi(s)) d s, \quad g_{1 / 2}(r)=\int_{0}^{r} \sqrt{g^{\prime}(s)} d s, \quad r \in \mathbb{R} .
$$

From the Rademacher theorem [Evans and Gariepy 1992], $g^{\prime}$ is a bounded Borelian representative of the derivative of $g$ in its class.

For all $\lambda>0$, the solution $v_{\lambda}$ of $\left(P_{\lambda}\right)$ associated to $w_{\lambda}$ has the following properties:

(i) Estimations using entropy inequations (for each entropy function $g$ ):

$$
\frac{\partial}{\partial t} G_{\Phi}\left(v_{\lambda}\right) \leq \operatorname{div}\left(g\left(w_{\lambda}\right) \nabla w_{\lambda}\right)-g^{\prime}\left(w_{\lambda}\right)\left|\nabla w_{\lambda}\right|^{2} \quad \text { in } Q .
$$

${ }^{5}$ According to an observation of [Evans and Portilheiro 2004].

${ }^{6}$ Thanks to the Cauchy-Lipschitz-Picard theorem via a first-order differential equation with given initial condition. 
Using the function $G$ defined in (8), the last inequality can be rewritten as

$$
\frac{\partial}{\partial t} G_{\Phi}\left(v_{\lambda}\right) \leq \Delta\left(G\left(w_{\lambda}\right)\right)-G^{\prime \prime}\left(w_{\lambda}\right)\left|\nabla w_{\lambda}\right|^{2} \text { in } Q .
$$

The following inequalities hold:

$$
\begin{gathered}
\int_{\Omega} G_{\Phi}\left(v_{\lambda}(t, x)\right) d x \leq \int_{\Omega} G_{\Phi}\left(v_{\lambda}(s, x)\right) d x \leq \int_{\Omega} G_{\Phi}\left(S_{w(0)}\right) d x, \quad t>s>0, \\
\left\|g_{\frac{1}{2}}\left(w_{\lambda}\right)\right\|_{L^{2}\left([0, T] ; H^{1}(\Omega)\right)}^{2} \stackrel{\text { def }}{=} \int_{Q} g^{\prime}\left(w_{\lambda}\right)\left|\nabla w_{\lambda}\right|^{2} d x d t \leq C_{g}, \quad C_{g}=C(g) .
\end{gathered}
$$

(ii) We have the following uniform a priori estimates:

$$
\begin{aligned}
\left\|v_{\lambda}\right\|_{L^{\infty}(Q)}+\left\|w_{\lambda}\right\|_{L^{\infty}(Q)} & \leq C_{1}, \\
\left\|w_{\lambda}\right\|_{L^{2}\left([0, T] ; H^{1}(\Omega)\right)}+\sqrt{\lambda}\left\|\frac{\partial v_{\lambda}}{\partial t}\right\|_{L^{2}(Q)} & \leq C_{2}, \\
\left\|\frac{\partial v_{\lambda}}{\partial t}\right\|_{L^{2}\left([0, T] ;\left(H^{1}(\Omega)\right)^{\prime}\right)} & \leq C_{3} .
\end{aligned}
$$

The frame constants depend on the extremum values of $\Phi$ and $S_{w(0)}$.

Proof. The general principle of the proof of this proposition may be found in [Evans 2004, p. 427]. This classical computation is somewhat akin to an entropy flux calculation for a hyperbolic conservation law, through choices of nondecreasing functions $g$ (see also [Gagneux and Millet 2015] for more details). We note that the inequality (9) is straightforward from the following relation, for any function $\Phi$ :

$$
\frac{\partial}{\partial t} G_{\Phi}\left(v_{\lambda}\right)-\operatorname{div}\left(g\left(w_{\lambda}\right) \nabla w_{\lambda}\right)=-g^{\prime}\left(w_{\lambda}\right)\left|\nabla w_{\lambda}\right|^{2}-\left(g\left(w_{\lambda}\right)-g\left(\Phi\left(v_{\lambda}\right)\right)\right) \frac{w_{\lambda}-\Phi\left(v_{\lambda}\right)}{\lambda}
$$

stated in [Evans and Portilheiro 2004; Evans 2004; Plotnikov 1996].

\section{Study of capillary effects}

4.1. Generalized "entropic" solutions. It follows from the uniform estimates of Proposition 1 that we can find subsequences $\left\{v_{\lambda_{k}}\right\}$ and $\left\{w_{\lambda_{k}}\right\}$ and a $\operatorname{pair}^{7}(v, w)$ such that, as $\lambda_{k} \rightarrow 0$,

$$
\begin{aligned}
v_{\lambda_{k}} \rightarrow v & \text { in } L^{\infty}(Q) \text { weakly-*, } \\
\frac{\partial v_{\lambda_{k}}}{\partial t} \rightarrow \frac{\partial v_{\lambda}}{\partial t} & \text { in } L^{2}\left([0, T] ;\left(H^{1}(\Omega)\right)^{\prime}\right) \text { weakly, } \\
w_{\lambda_{k}} \rightarrow w & \text { in } L^{\infty}(Q) \text { weakly- } * \text { and in } L^{2}\left([0, T] ; H^{1}(\Omega)\right) \text { weakly, }
\end{aligned}
$$

\footnotetext{
${ }^{7}$ A vanishing viscosity limit.
} 
and

$$
w_{\lambda_{k}}-\Phi\left(v_{\lambda_{k}}\right) \rightarrow 0 \text { in } L^{p}(Q) \text { strongly, for any finite } p .
$$

Furthermore, we can assume that ${ }^{8}$

$$
v_{\lambda_{k}} \rightarrow v \text { in } C^{0}\left([0, T] ; H^{1}(\Omega)^{\prime}\right) \text { strongly. }
$$

The associated Cauchy condition is given by

$$
\begin{cases}v(0, \cdot)=S_{w(0)} & \text { in } H^{1}(\Omega)^{\prime}, \text { a.e. in } \Omega \\ 0 \leq v(t, \cdot) \leq 1 & \text { in } \Omega, t>0 \\ \int_{\Omega} v(t, x) d x=\int_{\Omega} S_{w(0)}(x) d x, & t>0 .\end{cases}
$$

In addition, the pair $^{9}(v, w)$ belongs to the functional frame

$$
\left\{\begin{array}{l}
v \in L^{\infty}(Q) \cap C^{0}\left([0, T] ; H^{1}(\Omega)^{\prime}\right), \\
\frac{\partial v}{\partial t} \in L^{2}\left([0, T] ; H^{1}(\Omega)^{\prime}\right), \\
w \in L^{\infty}(Q) \cap L^{2}\left([0, T] ; H^{1}(\Omega)\right),
\end{array}\right.
$$

and is a solution of the boundary value problem

$$
\begin{cases}\frac{\partial v}{\partial t}-\Delta w=0 & \text { in } \mathscr{D}^{\prime}(Q) \text { and } L^{2}\left([0, T] ; H^{1}(\Omega)^{\prime}\right) \\ \frac{\partial w}{\partial n}=0 & \text { on } \Sigma \\ v(0, \cdot)=S_{w(0)} & \text { a.e. in } \Omega .\end{cases}
$$

Because of the nonmonoticity of $\Phi$, the information (11)-(14) is not sufficient to conclude that $w=\Phi(v)$, as we will see in what follows.

Let us denote by $\Xi$ the complementary of the set of points of $\mathscr{L}^{d+1}$-approximate continuity of $v$ according to the rigorous definition of the shock wave [Evans and Gariepy 1992; Gagneux and Madaune-Tort 1995]. The set $\Xi$ is Borelian and $\mathscr{L}^{d+1}$ negligible because $v$ is in $L^{\infty}(Q)$.

Let us assume that $\Xi$ is a countable union of smooth hypersurfaces $\Xi^{i}$ of $\mathbb{R}^{d+1}$ which admit a unit normal vector $v^{i}=\left(v_{1}^{i}, \ldots, v_{d}^{i}, v_{d+1}^{i}\right)=\left(\tilde{v}^{i}, v_{d+1}^{i}\right)$.

Using the usual notations for jumps in hyperbolic scalar laws and for the Hausdorff measure $\mathscr{H}^{d}$, very informally, the pair $(v, w)$ satisfies the Rankine-Hugoniot and entropy conditions for all $i$, integrating by parts locally in a vicinity of a given transition interface via appropriate smooth functions with compact support:

$$
\begin{gathered}
v_{d+1}^{i}[v]=\tilde{v}^{i} \cdot[\nabla w] \quad \text { and } \quad[w]=0 \quad \mathscr{H}^{d} \text {-a.e. on } \Xi^{i} \\
v_{d+1}^{i}\left[G_{\Phi}(v)\right]-\tilde{v}^{i} \cdot[\nabla w] g(w) \leq 0 \quad \mathscr{H}^{d} \text {-a.e. on } \Xi^{i}
\end{gathered}
$$

${ }^{8}$ From a classical compactness result of J. A. Dubinskii [Lions 1969, pp. 141-142].

${ }^{9}$ The pair $(v, w)$ is called a generalized solution of the problem $\left(P_{\Phi}\right)$. 
Relation (15) may be written in the form

$$
v_{d+1}^{i}\left(\left[G_{\Phi}(v)\right]-g(w)[v]\right) \leq 0 \quad \mathscr{H}^{d} \text {-a.e. on } \Xi^{i}
$$

with the notations (8) for the definition of $g$ (entropy function) and $G_{\Phi}$. In this form, relation (16) will be very useful to highlight the further developments.

4.2. Associated hysteresis effects. The analysis of hysteresis effects relies on the following proposition:

Proposition 2. There exist three $\mathscr{L}^{d+1}$-measurable and bounded functions, $\Lambda_{0}, \Lambda_{1}$ and $\Lambda_{2}$, that are representative of the respective influence of the three branches of the graph of $\Phi^{-1}$ (in the sense of the set theory) through the functions $\beta_{0}, \beta_{1}$ and $\beta_{2}$. Moreover, we have

$$
0 \leq \Lambda_{i} \leq 1 \quad \text { and } \quad \sum_{i=0}^{2} \Lambda_{i}=1 \quad \mathscr{L}^{d+1} \text {-a.e. in } Q .
$$

In addition, when $\lambda_{k}$ tends to $0^{+}$,

$$
\mu\left(v_{\lambda_{k}}\right) \text { converges to } \sum_{i=0}^{2} \Lambda_{i} \mu\left(\beta_{i}(w)\right) \text { in } L^{\infty}(Q) \text { weakly-* }
$$

for any numerical continuous function $\mu$. In addition, we have the following strong convergences:

$w_{\lambda_{k}}$ and $\Phi\left(v_{\lambda_{k}}\right)$ converge to $w$ in $L^{p}(Q)$ strongly for any finite $p$.

Finally, for any Lipschitz nondecreasing function $g$, we have the entropy relation in the sense of the measures in $Q$

$$
\frac{\partial}{\partial t}\left(\sum_{i=0}^{2} \Lambda_{i} G_{\Phi}\left(\beta_{i}(w)\right)\right) \leq \operatorname{div}(g(w) \nabla w)-g^{\prime}(w)|\nabla w|^{2} \quad \text { in } Q .
$$

Proof. The difficult technical proof of this proposition is not detailed here and can be found in [Evans and Portilheiro 2004; Evans 2004; Plotnikov 1996; 1994] with some adjustments.

When $\Lambda_{0}$ is equal to zero everywhere ${ }^{10}$ the method provides a response corresponding to the initial problem, thanks to the information contained in the complementary entropy relation (17). That is the main goal of the following proposition, based on the complementary information on the entropy given by (15)-(16), which enables us to determine the sense of circulation of the hysteresis loop.

${ }^{10}$ That corresponds in the final result to the neutralization of the decreasing part of the cubic introduced artificially to create a repulsive region. 
Proposition 3. In the framework of Proposition 2, we assume that

$$
\Lambda_{0}=0 \quad \text { in } Q, \quad \Lambda_{1}=1 \quad \text { in } Q_{1} \quad \text { and } \quad \Lambda_{2}=1 \quad \text { in } Q_{2} \text {, }
$$

where $Q_{1}$ and $Q_{2}$ are two open subsets of the cylinder $Q$, with a Lipschitz interface $\Sigma_{1,2}=\bar{Q}_{1} \cap \bar{Q}_{2}$ admitting a unit normal vector $v=\left(v_{1, \ldots}, v_{d}, v_{d+1}\right)$ of $\mathbb{R}^{d} \times \mathbb{R}$, oriented into $Q_{1}$.

Using the notations of Figure 2, the problem can be written more precisely as a problem with free surface:

$$
\begin{cases}v=\beta_{1}(w) \text { and } \frac{\partial v}{\partial t}-\Delta \Phi_{1}(v)=0 & \text { in } Q_{1}, \\ v=\beta_{2}(w) \text { and } \frac{\partial v}{\partial t}-\Delta \Phi_{2}(v)=0 & \text { in } Q_{2} .\end{cases}
$$

As a consequence of the information contained in the Rankine-Hugoniot and entropy relations (15)-(16), which are justified here along the shock wave $\Sigma_{1,2}$, the sign of the component $v_{d+1}$ of the normal vector $v$, i.e., its orientation during the time, is specified by the relations

$$
\begin{cases}v_{d+1}=0 & \text { if } A<w<B \\ v_{d+1} \geq 0 & \text { if } w=A \\ v_{d+1} \leq 0 & \text { if } w=B\end{cases}
$$

4.3. Interpretation of the results. According to Proposition 3, the expected hysteresis effect is well described by the pair $(v, w)$, the generalized solution of $\left(P_{\Phi}\right)$. The change of the expression of the state law, which governs the diffusion process according to the values of the reduced saturation, is given by (18). Moreover, relation (19) reveals that the interface $\Sigma_{1,2}$ evolves only if $w$ takes the value $A$ or $B$ (see Figure 1).

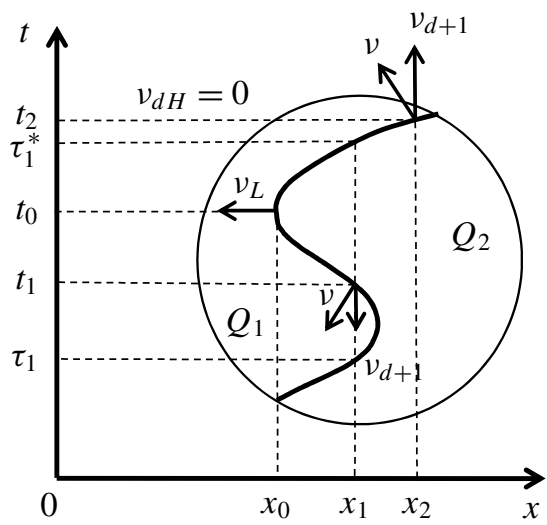

Figure 3. More general case illustrating Proposition 3. 


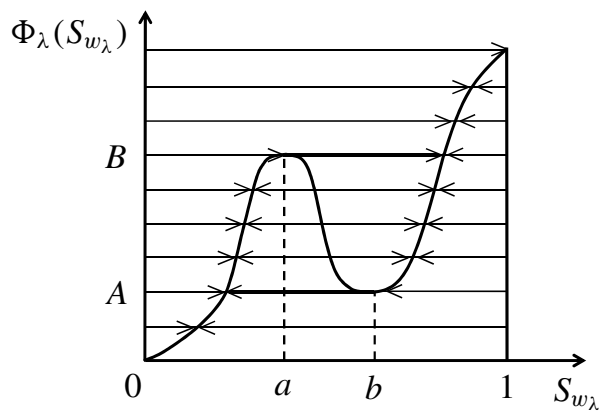

Figure 4. Flow of the auxiliary ordinary differential equation and emergence of the hysteresis effects when $\lambda \rightarrow 0$.

To illustrate the resulting hysteretic behavior, let us consider the generic example of Figure 3 on the previous page, zooming on a time interval representing three possible states of the point $x_{1}$ at three different times. We focus on the possible states corresponding to the abscissa $x_{1}$.

At the point $\left(t_{1}, x_{1}\right)$, we have a jump from $Q_{1}$ to $Q_{2}, w=B$, and we are in the imbibition phase (see also Figure 1). On the contrary, at the point $\left(t_{2}, x_{2}\right)$, we have a jump from $Q_{2}$ to $Q_{1}, w=A$, and we are in the drainage phase.

Therefore, the entropy method linked to the Sobolev regularization leads to a hysteresis loop similar to that obtained for Stefan's supercooling problem [Evans 2004]. The flow of the auxiliary ordinary differential equation (3) leads to hysteresis effects when $\lambda \rightarrow 0$ (Figure 4).

\section{Conclusion}

The hysteresis phenomena of flows in unsaturated porous media has been modeled with success, using the artificial introduction of an unstable spinodal interval and on a dynamic regularization process of Sobolev type.

\section{References}

[Aubin and Cellina 1984] J.-P. Aubin and A. Cellina, Differential inclusions: set-valued maps and viability theory, Grundlehren der Math. Wissenschaften 264, Springer, Berlin, 1984.

[Evans 2004] L. C. Evans, "A survey of entropy methods for partial differential equations", Bull. Amer. Math. Soc. (N.S.) 41:4 (2004), 409-438.

[Evans and Gariepy 1992] L. C. Evans and R. F. Gariepy, Measure theory and fine properties of functions, CRC Press, Boca Raton, FL, 1992.

[Evans and Portilheiro 2004] L. C. Evans and M. Portilheiro, "Irreversibility and hysteresis for a forward-backward diffusion equation”, Math. Models Methods Appl. Sci. 14:11 (2004), 1599-1620.

[Gagneux and Madaune-Tort 1995] G. Gagneux and M. Madaune-Tort, Analyse mathématique de modèles non linéaires de l'ingénierie pétrolière, Mathématiques \& Applications 22, Springer, Berlin, 1995. 
[Gagneux and Millet 2015] G. Gagneux and O. Millet, "A model for capillary hysteresis loops in unsatured porous media theory", 2015. Submitted to Math. Mech. Solids.

[Lions 1969] J.-L. Lions, Quelques méthodes de résolution des problèmes aux limites non linéaires, Dunod, Paris, 1969.

[Lions and Magenes 1968] J.-L. Lions and E. Magenes, Problèmes aux limites non homogènes et applications, I, Travaux et Recherches Mathématiques 17, Dunod, Paris, 1968.

[Plotnikov 1994] P. I. Plotnikov, "Passage to the limit with respect to viscosity in an equation with a variable direction of parabolicity", Differentsial' nye Uravneniya 30:4 (1994), 665-674, 734. In Russian; translated in Differential Equations 30:4 (1994), 614-622.

[Plotnikov 1996] P. I. Plotnikov, "Forward-backward parabolic equations and hysteresis", pp. 183209, 257-258 in Boundary-value problems of mathematical physics and related problems of function theory, XXVII, Zap. Nauchn. Sem. S.-Peterburg. Otdel. Mat. Inst. Steklov. (POMI) 233, 1996. Reprinted in J. Math. Sci. 93:5 (1999), 747-766.

[Smarrazzo 2008] F. Smarrazzo, "On a class of equations with variable parabolicity direction", Discrete Contin. Dyn. Syst. 22:3 (2008), 729-758.

[Smarrazzo and Tesei 2010] F. Smarrazzo and A. Tesei, "Long-time behavior of solutions to a class of forward-backward parabolic equations", SIAM J. Math. Anal. 42:3 (2010), 1046-1093.

[Smarrazzo and Tesei 2012] F. Smarrazzo and A. Tesei, "Degenerate regularization of forwardbackward parabolic equations: the regularized problem", Arch. Ration. Mech. Anal. 204:1 (2012), 85-139.

Received 10 Sep 2015. Revised 3 Dec 2015. Accepted 15 Jan 2016.

GÉRARD GAGNEUX: gerard.maryse.gagneux@gmail.com

Laboratoire des Sciences de l'Ingénieur pour l'Environnement, UMR-CNRS 7356,

Université de La Rochelle, avenue Michel Crépeau, 17042 La Rochelle Cedex 1, France

OLIVIER MILLET: olivier.millet@univ-lr.fr

Laboratoire des Sciences de l'Ingénieur pour l'Environnement, UMR-CNRS 7356,

Université de La Rochelle, avenue Michel Crépeau, 17042 La Rochelle Cedex 1, France

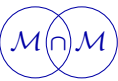


EDITORIAL BOARD

ANTONIO CARCATERRA

ERIC A. CARLEN

FRANCESCO DELL'ISOLA

RAFFAELE ESPOSITO

ALBERT FANNJIANG

Gilles A. FranCFORT

Pierangelo MARCATI

JEAN-JACQUES MARIGO

PETER A. MARKOWICH

MARTIN OSTOJA-STARZEWSKI

PIERRE SEPPECHER

DAVID J. STEIGMANN

PAUl STEINMANN

PierRe M. SuQueT

MANAGING EDITORS

MICOL AMAR

CORRADO LATTANZIO

ANGELA MADEO

MARTIN OSTOJA-STARZEWSKI

ADVISORY BOARD

ADNAN AKAY

Holm AltenBaCH

MICOL AMAR

HARM ASKES

TEODOR ATANACKOVIĆ

VICTOR BERDICHEVSKY

GUY BOUCHITTÉ

ANDREA BRAIDES

ROBERTO CAMASSA

MAURO CARFORE

ERIC DARVE

FELIX DARVE

ANNA DE MASI

GianPiEtro DEL Piero

EMMANUELE Di BENEDETTO

BERNOLD FIEDLER

IRENE M. GAMBA

DAVID Y. GAO

SERGEY GAVRILYUK

TIMOTHY J. HEALEY

DOMINIQUE JEULIN

ROGER E. KHAYAT

CORRADO LATTANZIO

ROBERT P. LIPTON

ANGELO LUONGO

ANGELA MADEO

JUAN J. MANFREDI

CARLO MARCHIORO

GÉRARD A. MAUGIN

ROBERTO NATALINI PATRIZIO NEFF

ANDREY PIATNITSKI

ERRICO PRESUTTI

MARIO PULVIRENTI

LUCIO RUSSO

Miguel A. F. SANJUAN

PATRICK SElVADURAI

ALEXANDER P. SEYRANIAN

MIROSLAV ŠILHAVÝ

GUIDO SWEERS

ANTOINETTE TORDESILLAS

LEV TRUSKINOVSKY

JUAN J. L. VELÁZQUEZ VINCENZO VESPRI ANGELO VULPIANI msp.org/memocs

Università di Roma "La Sapienza", Italia

Rutgers University, USA

(CO-CHAIR) Università di Roma "La Sapienza", Italia

(TREASURER) Università dell'Aquila, Italia

University of California at Davis, USA

(CO-CHAIR) Université Paris-Nord, France

Università dell'Aquila, Italy

École Polytechnique, France

DAMTP Cambridge, UK, and University of Vienna, Austria

(CHAIR MANAGING EDITOR) Univ. of Illinois at Urbana-Champaign, USA

Université du Sud Toulon-Var, France

University of California at Berkeley, USA

Universität Erlangen-Nürnberg, Germany

LMA CNRS Marseille, France

Università di Roma "La Sapienza", Italia

Università dell'Aquila, Italy

Université de Lyon-INSA (Institut National des Sciences Appliquées), France

(CHAIR MANAGING EDITOR) Univ. of Illinois at Urbana-Champaign, USA

Carnegie Mellon University, USA, and Bilkent University, Turkey

Otto-von-Guericke-Universität Magdeburg, Germany

Università di Roma "La Sapienza", Italia

University of Sheffield, UK

University of Novi Sad, Serbia

Wayne State University, USA

Université du Sud Toulon-Var, France

Università di Roma Tor Vergata, Italia

University of North Carolina at Chapel Hill, USA

Università di Pavia, Italia

Stanford University, USA

Institut Polytechnique de Grenoble, France

Università dell'Aquila, Italia

Università di Ferrara and International Research Center MEMOCS, Italia

Vanderbilt University, USA

Freie Universität Berlin, Germany

University of Texas at Austin, USA

Federation University and Australian National University, Australia

Université Aix-Marseille, France

Cornell University, USA

École des Mines, France

University of Western Ontario, Canada

Università dell' Aquila, Italy

Louisiana State University, USA

Università dell'Aquila, Italia

Université de Lyon-INSA (Institut National des Sciences Appliquées), France University of Pittsburgh, USA

Università di Roma "La Sapienza”, Italia

Université Paris VI, France

Istituto per le Applicazioni del Calcolo "M. Picone", Italy

Universität Duisburg-Essen, Germany

Narvik University College, Norway, Russia

Università di Roma Tor Vergata, Italy

Università di Roma "La Sapienza”, Italia

Università di Roma “Tor Vergata”, Italia

Universidad Rey Juan Carlos, Madrid, Spain

McGill University, Canada

Moscow State Lomonosov University, Russia

Academy of Sciences of the Czech Republic

Universität zu Köln, Germany

University of Melbourne, Australia

École Polytechnique, France

Bonn University, Germany

Università di Firenze, Italia

Università di Roma La Sapienza, Italia

MEMOCS (ISSN 2325-3444 electronic, 2326-7186 printed) is a journal of the International Research Center for the Mathematics and Mechanics of Complex Systems at the Università dell'Aquila, Italy.

Cover image: "Tangle” by $\odot$ John Horigan; produced using the Context Free program (contextfreeart.org).

PUBLISHED BY

7 mathematical sciences publishers

nonprofit scientific publishing

http://msp.org/

(C) 2016 Mathematical Sciences Publishers 
Mathematics and Mechanics of Complex Systems vol. 4 no. 1

Gradient materials with internal constraints

Albrecht Bertram and Rainer Glüge

Unified geometric formulation of material uniformity and evolution

Marcelo Epstein and Manuel de León

Electromechanics of polarized lipid bilayers

David J. Steigmann and Ashutosh Agrawal

Orthogonal polynomials and Riesz bases applied to the solution of Love's equation

Pierluigi Vellucci and Alberto Maria Bersani

Modeling capillary hysteresis in unsatured porous media

Gérard Gagneux and Olivier Millet

Discrete double-porosity models for spin systems

Andrea Braides, Valeria Chiadò Piat and Margherita Solci

Correction to "On the theory of diffusion and swelling in finitely deforming elastomers"

Gary J. Templet and David J. Steigmann

MEMOCS is a journal of the International Research Center for the Mathematics and Mechanics of Complex Systems at the Università dell' Aquila, Italy.

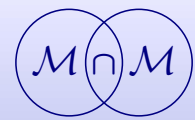

